\title{
An Integrated approach for color Image Segmentation using edge and color information
}

\author{
M.Mary Synthuja Jain Preetha ${ }^{1}$, L.Padma Suresh ${ }^{2}$ \\ ${ }^{I}$ (ECE, Noorul Islam Centre for Higher Education, Kumaracoil,India) \\ 2 (Professor/EEE, Noorul Islam Centre for Higher Education, Kumaracoil,India)
}

\begin{abstract}
Image segmentation is a fundamental and complex task in many image processing applications. In this paper, we propose a hybrid method for color image segmentation by integrating color edge information and seeded region growing. We use the color edge information to decide the best position to place the seed and to control the growth of the region. By using color edge detection technique, regions without edges are labeled as the initial seed. Regions are grown from this seed point by adding neighboring pixels that are similar based on homogeneity criterion. The obtained edge map and the region growth map are fused together to produce homogeneous regions with closed boundaries. Finally region merging is done to merge small regions.
\end{abstract}

Keywords: Seeded Region Growing, Color Similarity, Edge detection, Region Merging

\section{Introduction}

Image Segmentation is a significant and a very complex technique in image processing. To dig out the useful information from any image, the foreground and background must be separated. In image processing segmentation is considered as the right procedure and practice for this task. The automatic image segmentation technique can be classified as 1) Segmentation based on thresholding 2) Boundary-Based Segmentation 3) Region-Based Segmentation 4) Hybrid techniques.

Many segmentation algorithms have been proposed in the literature where edge, color and texture were considered as the basic metric for segmentation. Image segmentation is used in various applications like medical imaging, recognition, detection and many more. Many segmentation algorithms have been implemented to segment gray scale images[1]. Segmentation based on thresholding is based on the assumption that pixels in the particular image may be either a background or foreground. Usually this type of segmentation is used to segment gray scale images [2] - [4]. Due to the advancement in color image processing, color image segmentation becomes essential to extract meaningful segments from images. Boundary based segmentation can be used to segment color images. This type of segmentation is based on the assumption that there will be an abrupt change in the pixel properties (intensity, color, texture) between different regions [6] - [8]. Region based segmentation is based on the assumption that neighboring pixels within a region should have similar pixel properties like color, texture and intensity [9] - [12]. Hybrid methods combine boundary based and region based segmentation to obtain better segmentation result [13] - [18].

\subsection{Seeded Region Growing}

Segmentation of images can be done based on similarities or discontinuities between pixels. Region based segmentation is carried out based on the similarities in the given image. Region based segmentation create regions directly by grouping pixels together which share common features. The regions that are formed using region based segmentation have the following properties.

i) The entire image $\mathrm{R}$ can be divided into several subregions $\mathrm{R} 1, \mathrm{R} 2, \ldots \ldots ., \mathrm{Rn}$.

ii) Each region Ri should be connected based on some predicate (ie) Uniformity predicate is true.

iii) Two different regions say $\mathrm{Ri}$ and $\mathrm{Rj}$ should be distinct (ie) Uniformity predicate is false.

In this paper, we propose a new color image segmentation algorithm based on seeded region growing that automatically 1) selects initial seed for the color images using edge information in the CIE $L * a * b$ color space 2) Perform region growing based on the color similarity. 3) perform region merging based on the size of segmented regions.

Using color edge detection, our algorithm first detects the edges, which locate the regions without edges. The pixels present in each distinct region receive a label and the pixels with same label are referred as seed. These seeds will grow into the higher edge density area. Region growing is done based on the color similarity between the seed and the adjacent pixel.

Our algorithm is compiled in a MatLab environment and tested over images obtained from the Berkeley database. 


\section{Proposed Work}

Fig(1) shows the general block diagram of our proposed algorithm. Initially the RGB color image is transformed to CIE L*a*b color space. Though many color spaces like HIS, HSV, YCbCr etc., are present the reason for choosing CIE L*a*b for our work is, the color difference which we see correspond to the distances when measured colorimetrically (ie) it approximates human vision. The L component closely matches human perception of lightness.

The proposed algorithm uses a color edge detection algorithm, that identifies the regions into which the image to be segmented. It also provides the direction for the growth procedure.

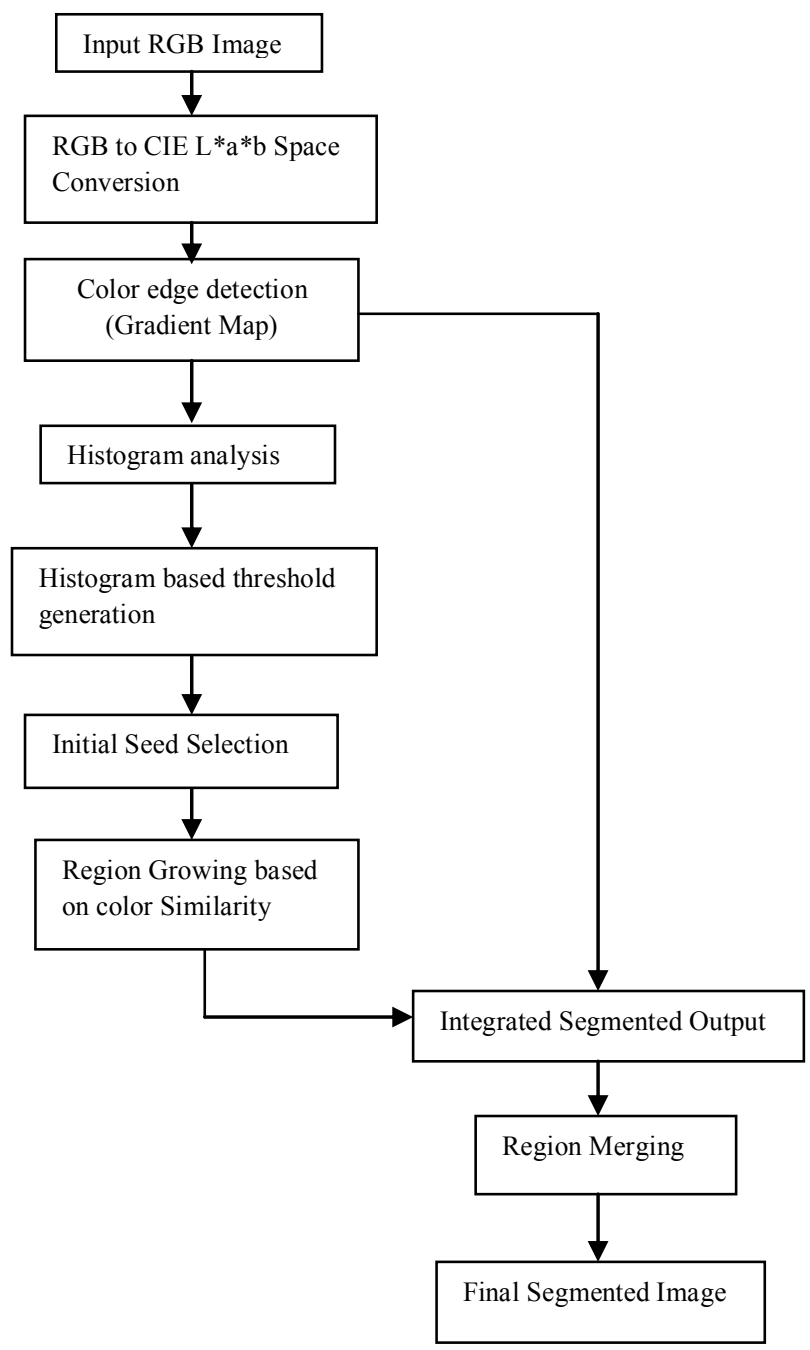

\subsection{Edge detection}

Fig 1: General Block diagram of Proposed Work

Variation of scene features such as color, brightness, texture gives rise to edges. Detecting edges will detect the discontinuities of the scene intensity function. Detecting edges will provide the basic shape information. Edges are locations with high image gradient or derivative. Edges are vector variables which gives the magnitude of the gradient and direction. Edge detection methods can be categorized as: 1) first order derivative/Gradient Methods 2) Second derivative methods 3)Optimal Edge detector. Gradient operators such as Roberts, Prewitt and Sobel operators [21]-[23] detect edges by finding the magnitude in its first derivative. The second derivative operators such as Laplacian operator, detect edges by searching for Zero-Crossings in the second derivative. The edge detection procedure using the magnitude of the gradient as defined by Lee and Cok [20], for the 3D image is described as,

Let $\mathrm{x}, \mathrm{y}, \mathrm{z}$ denote three color channels and i,j denote the spatial co-ordinates of a pixel. The following variables are defined to find the corresponding gradient values in each location:

$$
\begin{gathered}
q=\left(\frac{d x}{d i}\right)^{2}+\left(\frac{d y}{d i}\right)^{2}+\left(\frac{d z}{d i}\right)^{2} \\
r=\left(\frac{d x}{d i} \frac{d x}{d j}\right)+\left(\frac{d y}{d i} \frac{d y}{d j}\right)+\left(\frac{d z}{d i} \frac{d z}{d j}\right)
\end{gathered}
$$




$$
s=\left(\frac{d x}{d j}\right)^{2}+\left(\frac{d y}{d j}\right)^{2}+\left(\frac{d z}{d j}\right)^{2}
$$

The gradient matrix $\mathrm{D}$ for the vector field $\mathrm{f}$ can be defined as

$$
D=\left[\begin{array}{ll}
\frac{\mathrm{dx}}{\mathrm{di}} & \frac{d x}{d j} \\
\frac{d y}{d i} & \frac{d y}{d j} \\
\frac{d z}{d i} & \frac{d z}{d j}
\end{array}\right]
$$

The distance from a given point with a unit vector $\mathrm{u}$ in the spatial domain $d=\sqrt{u^{T} D^{T} D u}$ corresponds to the distance travelled in color domain. The largest eigen value of the matrix $D^{T} D$ gives the maximum distance travelled. The matrix $D^{T} D$ is given by

$$
D^{T} D=\left[\begin{array}{ll}
q & r \\
r & s
\end{array}\right]
$$

The largest eigen value $\lambda$ is $\lambda=\frac{1}{2}\left(q+s+\sqrt{(q+h)^{2}-4\left(q s-t^{2}\right)}\right)$. The gradient value in each location is $G=\sqrt{\lambda}$.

\subsection{Initial seed selection}

Region growing is a procedure in which pixels are grouped together to form regions based on some similarity conditions like color, gray level, texture or pixel intensity. Growing process is initiated by selecting an initial pixel called as seed pixel which acts as a seed for the growing process. The quality of final segmentation is highly dependent on the selection of seed pixel. In our proposed work, the seed selection procedure finds regions where edge map displays no edge. The selected regions are the initial seed.

\subsection{Threshold Generation}

From the CIE L*a*b color space, the gradient values are calculated as explained in section I.A .Fig.2. From the histogram plot of the gradient values initial threshold is generated. For images in which a large percentage of gradient values spread over a narrow range, a high threshold is chosen and for images in which large percentage of gradient values spread over a wide range, a low threshold value is chosen[19]. The threshold T is chosen in such a manner that all low gradient regions are taken as the initial seed. As given in GSEG algorithm [19], we choose 5 as the low threshold value and 10 as the high threshold value. Fig.4 Initial seeds are generated by sensing all regions whose gradient value is less than the chosen threshold. The selected seed should be large enough to prevent multiple seed generation within a region. The size of the seed will vary according to the size of the region. Each seed pixel will receive unique label and the collective set of all initial seed pixels is called a seed map. Fig.3 gives a sample labeled seed map.

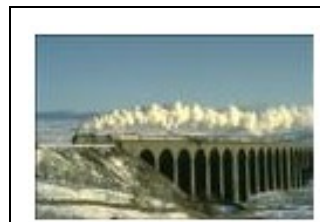

(a)

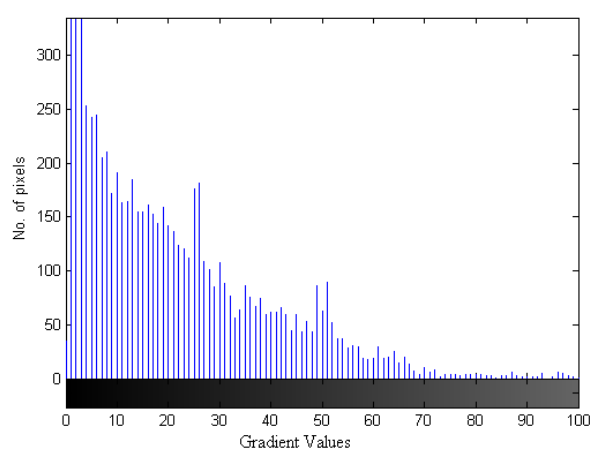

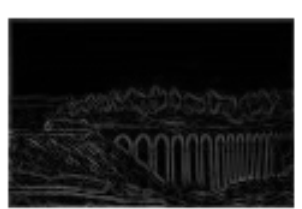

(b)

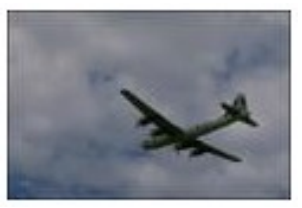

(d)

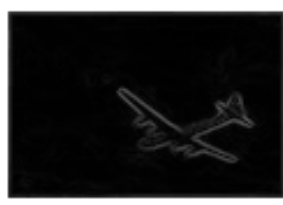

(e)

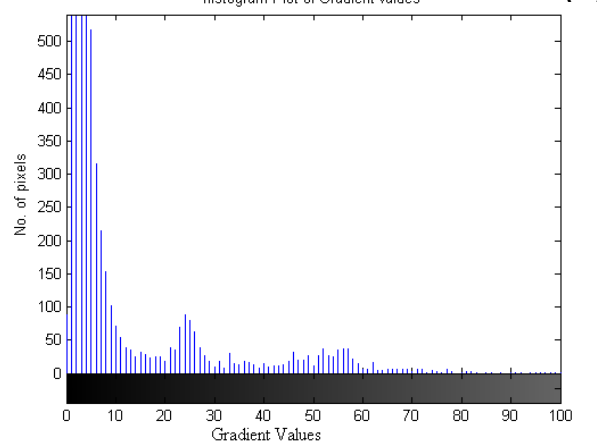

(f)

Fig. 2. Threshold generation (a) input Image (b) Gradient Map of (a) ,(c)(T = 10 case) gradient map histogram for input (a), (d) input Image (e) Gradient Map of (d), (f)( $(\mathrm{T}=5$ case) gradient map histogram for input (d) 


\section{Seeds Map (Labeled Image)}

\begin{tabular}{|l|l|l|l|l|l|l|l|}
\hline $\mathbf{1}$ & $\mathbf{1}$ & 0 & 0 & $\mathbf{3}$ & $\mathbf{3}$ & 0 & 0 \\
\hline $\mathbf{1}$ & $\mathbf{1}$ & 0 & 0 & 0 & $\mathbf{3}$ & $\mathbf{3}$ & $\mathbf{3}$ \\
\hline $\mathbf{1}$ & $\mathbf{1}$ & $\mathbf{1}$ & 0 & $\mathbf{3}$ & $\mathbf{3}$ & $\mathbf{3}$ & $\mathbf{3}$ \\
\hline 0 & 0 & $\mathbf{1}$ & 0 & 0 & $\mathbf{3}$ & $\mathbf{3}$ & 0 \\
\hline $\mathbf{2}$ & 0 & 0 & 0 & 0 & 0 & 0 & 0 \\
\hline $\mathbf{2}$ & $\mathbf{2}$ & $\mathbf{2}$ & 0 & $\mathbf{4}$ & $\mathbf{4}$ & $\mathbf{4}$ & 0 \\
\hline 0 & $\mathbf{2}$ & $\mathbf{2}$ & 0 & 0 & $\mathbf{4}$ & $\mathbf{4}$ & $\mathbf{4}$ \\
\hline 0 & $\mathbf{2}$ & 0 & 0 & 0 & 0 & $\mathbf{4}$ & $\mathbf{4}$ \\
\hline
\end{tabular}

$\checkmark$\begin{tabular}{|l|l|}
\hline Seed Label & No. of Pixels \\
\hline 1 & 8 \\
\hline 2 & 7 \\
\hline 2 & 11 \\
\hline 3 & 8 \\
\hline 4 &
\end{tabular}

Fig 3: Labeled seed Map and number of pixels per seed

\section{Region Growing}

After the selection of initial seed, there are several pixels which are to be grouped to the seed pixel to form a large region. Let these pixels be named as ungrouped pixels and all the allocated pixels are termed as grouped pixels. We use the color similarity function as a predicate for region growing algorithm.

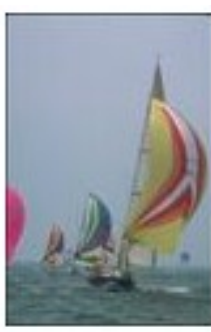

(a)

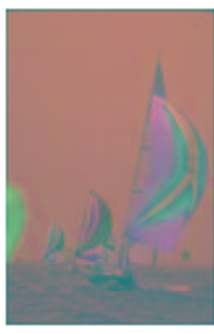

(b)

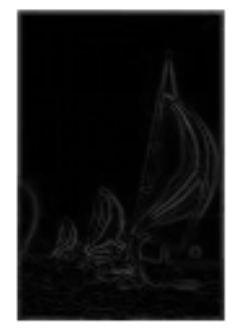

(c)

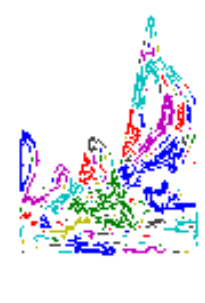

(d)

Fig 4. a) Input Image b)RGB to CIE L*a*b c) Gradient Map d) Initial seed

Let $\mathrm{P}_{\mathrm{s}}$ be the seed pixel and $\mathrm{P}_{\mathrm{i}}$ be any ungrouped pixel. The color similarity between the two pixels is calculated and the difference between the two pixels will show how far apart they are in the color space. If the difference between the two pixels is greater than a particular threshold it is discarded and if it is less than the threshold it is grouped with the seed pixel.

Since we are using the CIE L*a*b color space, the color similarity is calculated as,

$$
\begin{aligned}
& \Delta L=L_{s}-L_{i} \\
& \Delta a=a_{s}-a_{i} \\
& \Delta b=b_{s}-b_{i}
\end{aligned}
$$

where $L_{s}, a_{s}$, and $b_{s}$ gives the $\mathrm{L}$ channel, a channel and $\mathrm{b}$ channel values of the seed pixel and $L_{i}, a_{i}$, and $b_{i}$ gives the $\mathrm{L}$ channel, a channel and $\mathrm{b}$ channel of any ungrouped pixel respectively. $\Delta L$ gives the lightness difference, $\Delta a$ gives the red/green difference and $\Delta b$ gives the yellow/blue difference. The CIE L*a*b coordinate system is shown in Table.1. The CIE L*a*b color difference between the pixels is shown in Table.2.

Table 1: CIE Lab Co-ordinates

\begin{tabular}{|l|l|}
\hline L & Light - Dark \\
\hline $\mathrm{a}$ & Red - Green \\
\hline $\mathrm{b}$ & Yellow - Blue \\
\hline
\end{tabular}

Table 2: CIE Lab Color Difference

\begin{tabular}{|l|l|l|}
\hline & \multicolumn{1}{|c|}{+} & \multicolumn{1}{c|}{-} \\
\hline$\Delta \mathrm{L}$ & Light & Dark \\
\hline$\Delta \mathrm{a}$ & Red & Green \\
\hline$\Delta \mathrm{b}$ & Yellow & Blue \\
\hline
\end{tabular}

The total color difference $\Delta T=\sqrt{\Delta L^{2}+\Delta a^{2}+\Delta b^{2}}$. The seeded region growing procedure is repeated until all the ungrouped pixels in the image have been allocated to its corresponding region. In our algorithm we choose 2 as the threshold for color similarity. 
The detected color edges and the resultant seeded region grown images are integrated to provide a better segmentation result. The boundary pixels of each homogeneous region and the edge pixels are first extracted. Pixels which are detected both as boundary and edge pixel will become a boundary pixel of a region. The pixel which is detected as an edge pixel but not as a boundary should become a pixel on the final region boundary. The pixel which is detected as a boundary but not as an edge should be the pixel inside a region. These three possibilities are explained by means of Table. 3 .

Table 3: Integrating boundary and edge pixels

\begin{tabular}{|c|c|l|}
\hline \multicolumn{2}{|c|}{ Extracted Pixel } & \multirow{2}{*}{ Result } \\
\cline { 1 - 2 } Boundary & Edge & \\
\hline 1 & 1 & Final Boundary \\
\hline 0 & 1 & Final Boundary \\
\hline 1 & 0 & Region \\
\hline
\end{tabular}

\subsection{Region Merging}

During initial seed selection several seeds were generated, which splits a region into several small regions. To prevail over this oversegmentation problem, we perform region merging. In our proposed work we merge two regions based on the size. The distance between two regions is computed and if this distance is less than a particular threshold, we merge the two regions. Again the distance between this newly generated region and its adjacent region is computed and merging is done based on the chosen threshold. This process is repeated until no region has a distance less than the threshold. In our experiment, regions less than $0.65 \%$ of the image are merged to form a large homogeneous region.

\section{Results and Discussion}

We have performed the experiment using our proposed segmentation algorithm in the color images taken from the publically available Berkeley segmentation dataset. We can able to obtain reasonably good results. The results of our proposed algorithm at different stages are presented in fig 4(a) - (f).

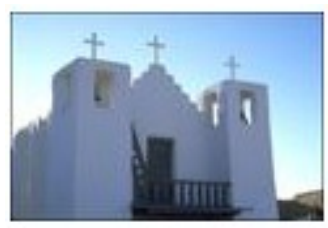

(a)

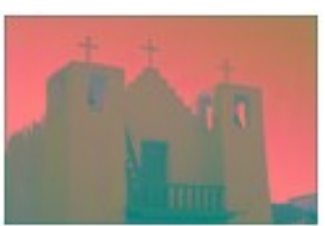

(b)

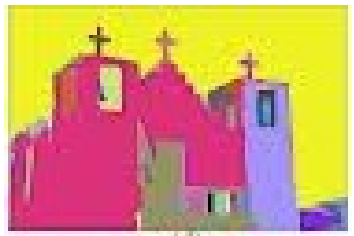

(e)

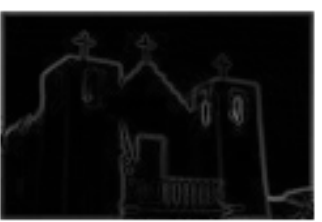

(c)

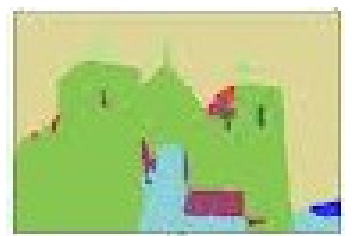

(f)

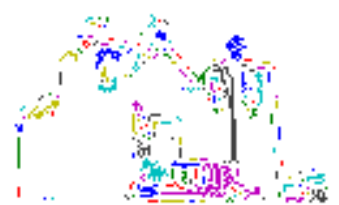

(d)

Fig.5. a) Input Image b)RGB to CIE L*a*b c) Gradient Map d) Initial seed e) Seeded Region growing fused with edge map f)Segmented Image after Region Merging

The input RGB image is shown in fig 5(a) and its CIE L*a*b space image is shown in fig 5(b). The edge map using gradient values and the initial seed map is shown in fig 5(c) and 5(d) respectively. The segmented image based on color similarity and edge map is given in fig 5(e). The final segmented image after region merging based on the size is shown in fig 5(f). Fig (6) and(7) provides additional results for our proposed algorithm. 


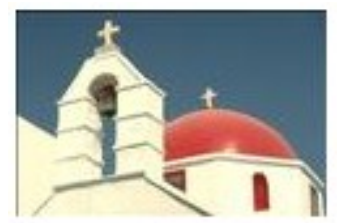

(a)

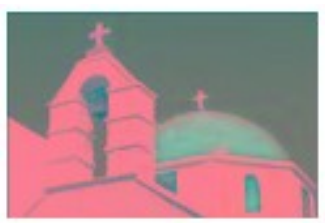

(b)

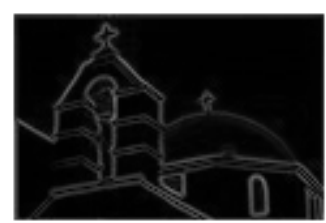

(c)

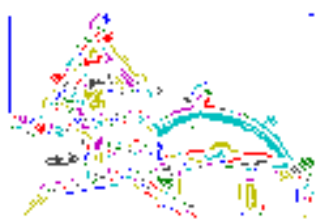

(d)

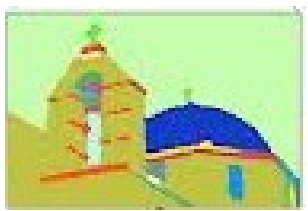

(e)

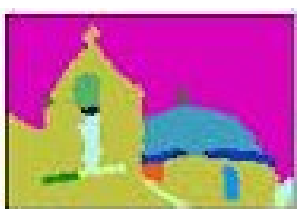

(f)

Fig.6. a) Input Image b)RGB to CIE L*a*b, c) Gradient Map d) Initial seed e) Seeded Region growing fused with edge map f)Segmented Image after region Merging

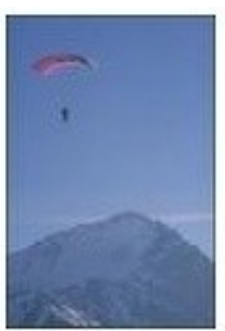

(a)

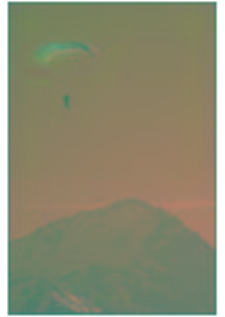

(b)

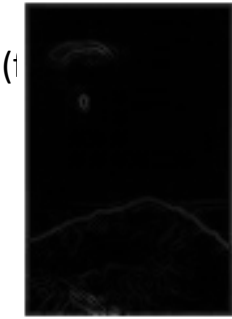

(c)

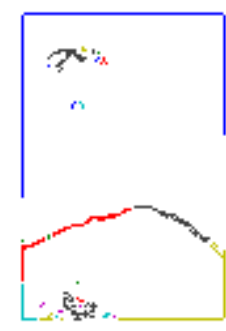

(d)

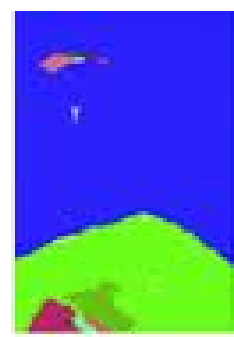

(e)

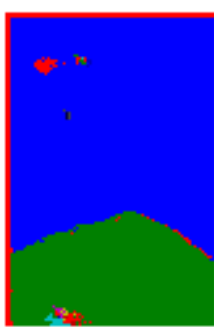

(f)

Fig.7. a) Input Image b)RGB to CIE L*a*b, c) Gradient Map d) Initial seed e) Seeded Region growing fused with edge map f)Segmented Image after region Merging

\section{Conclusion}

In this paper, we proposed an algorithm for color image segmentation based on seeded region growing. Our work is primarily based on color edge detection and seeded region growing. Segmenting images based on color will fail to identify meaningful objects. Segmentation based on edge detection fails to extract connected edges. Our work uses both color and edge information to produce more meaningful regions. Experimental results show that our algorithm can produce reasonably good results in various color images.

\section{References}

[1] H. Cheng, X. Jiang, Y. Sun, and J. Wang, “Color image segmentation:Advances \& prospects,” Pattern Recognition., 34( 12), 2001, 2259-2281.

[2] N. Otsu, A threshold selection method from gray-level histogram, IEEE Transactions on Systems, Man, and Cybernetics ,9(1), $1979,62-66$.

[3] A.K.C. Wong, P.K. Sahoo, A gray-level threshold selection method based on maximum entropy principle, IEEE Transactions on Systems, Man and Cybernetics, 19(4), 1989, 641-647.

[4] H.D. Cheng, C.H. Chen, H.H. Chiu, H. Xu, Fuzzy homogeneity approach to multilevel thresholding, IEEE Transactions on Image Processing ,7(7), 1998, 1084-1088.

[5] E. Saber, A. Tekalp, and G. Bozdagi, "Fusion of color and edge information for improved segmentation and edge linking," Imag. Vis.Comput., 15(10),1997, 769-780.

[6] J. Basak, B. Chanda, On edge and line linking with connectionist model, IEEE Transactions on System, Man, and Cybernetics ,24(3), 1994, 413-428.

[7] A. Marteli, An application of heuristic search methods to edge and contour detection, Graphics and Image Processing, 19(2), 1976, 73-83.

[8] V.S. Nalwa, E. Pauchon, Edgel aggregation and edge description, Computer Vision, Graphics, and Image Processing, 40(1), 1987, 79-94.

[9] A. Tremeau, N. Bolel, A region growing and merging algorithm to color segmentation, Pattern Recognition .30(7), 1997, 11911203.

[10] Frank Y.Shih, Shouxian Cheng, Automatic Seeded Region Growing for color image Segmentation, Pattern Recognition, 23, 2005, $877-886$.

[11] Luis Garcia Ugarriza, Eli Saber, Sreenath Rao Vantaram, Vincent Amuso, Mark Shaw, Ranjit Bhaskar, Automatic Image Segmentation by Dynamic Region Growth and Multiresolution Merging, 18(10), 2009, 2275-2009.

[12] S. Wan and W. Higgins, "Symmetric region growing," IEEE Trans.Image Process., 12(9), 2003, $1007-1015$. 
[13] J.Fan, David K.Y.Yau, Ahmed K.Elmagarmid, Walid G.Aref, Automatic Image Segmentation by Integrating Color-Edge Extraction and Seeded Region Growing, IEEE transaction on Image Processing, 10(10), 2001, 1454-1466.

[14] T. Pavlidis, Y.T. Liow, Integrating region growing and edge detection, IEEE Transactions on Pattern Analysis and Machine Intelligence ,12(3), 1990, 225-233.

[15] C. Chu, J.K. Aggarwal, The integration of image segmentation maps using region and edge information, IEEE Transactions on Pattern Analysis and Machine Intelligence,15(2), 1993, 1241-1252.

[16] J.F. Haddon, J.F. Boyce, Image segmentation by unifying region and boundary information, IEEE Transactions on Pattern Analysis and Machine Intelligence ,12(10), 1990, 929-948.

[17] J. Chen, T. Pappas, A. Mojsilovic, and B. Rogowitz, "Adaptive image segmentation based on color and texture," in Proc. Int. Conf. ImageProcess., 3, 2002, 777-780.

[18] J. Chen, T. Pappas, A. Mojsilovic, and B. Rogowitz, “Adaptive perceptual color-texture image segmentation," IEEE Trans. Image Process., 14(10), 2005, 1524-1536.

[19] Luis Garcia Ugarriza, Eli Saber, Sreenath Rao Vantaram, Vincent Amuso, Mark Shaw, andRanjit Bhaskar,” Automatic Image Segmentation by Dynamic Region Growth and Multiresolution Merging" IEEE Trans. Image Process., 18(10), 2009, 2275-2288.

[20] H. Lee and D. Cok, "Detecting boundaries in a vector field," IEEE Trans. Signal Process., 39(5), 1991, 1181-1194.

[21] J. Prewitt, "Object enhancement and extraction," in Picture Processing and Psychopictures, B. Lipkin and A. Rosenfeld, Eds. NewYork: Academic, 1970.

[22] L. G. Roberts, "Machine perception of three-dimensional solids," in Optical and Eletrooptical Information Processing, J. T. Tripperts,Ed. Cambridge, MA: MIT Press, 1965.

[23] L. Davis, "Survey of edge detection techniques, computer vision," Graph. Image Process., 4, 1975, $248-270$. 